\title{
Editorial
}

\section{Welcome to a New Journal-Journal of Fertility Preservation}

\author{
Christiani A. Amorim ${ }^{1}$ and Peter E. J. Bols ${ }^{2}$ \\ ${ }^{1}$ Pôle de Recherche en Gynécologie, Institut de Recherche Expérimentale et Clinique, Université Catholique de Louvain, 1200 Brussels, Belgium \\ ${ }^{2}$ Laboratory for Veterinary Physiology and Biochemistry, Gamete Research Center, Faculty of Pharmaceutical, Biomedical and Veterinary \\ Sciences, 2610 Wilrijk, Belgium \\ Address correspondence to ChristianiA. Amorim, christiani.amorim@uclouvain.be; PeterE.J.Bols, peter.bols@uantwerpen.be
}

Received 10 December 2019; Accepted 10 December 2019

Copyright $(\odot 2019$ Christiani A. Amorim and Peter E. J. Bols. This is an open access article distributed under the terms of the Creative Commons Attribution License, which permits unrestricted use, distribution, and reproduction in any medium, provided the original work is properly cited.

The medical subdiscipline of fertility preservation was born from the desire of human beings to have a proper child. Cancer patients undergoing treatments with gonadotoxic sideeffects leading to sterility or infertility and women and men with benign conditions that can negatively affect their quantity and/or quality of gametes have been the most important drivers for the development of this field. In addition, fertility preservation has been also propelled by different issues from healthy women and men. Delay of childbearing age for professional or personal reasons, new data on risks of fathering children into older ages for men and the adverse effects of gender affirming procedures on the reproductive potential of transgender people, and so on, have increased the development and application of fertility preservation strategies and the importance of this field in current times.

Moreover, fertility preservation is not only a concern in humans, as the need for the preservation of genetic material from endangered species, animals with important genetic traits or biobanking in general will also greatly benefit from the development of alternative preservation strategies which might be forced upon us by the inevitable consequences of environmental and climate change. In addition, to further optimize fertility preservation strategies, the use of animal in vitro models will certainly benefit progress in both the human and animal research fields. As a consequence, research results on "fertility preservation" at the level of an individual patient or specimen will be often translated into "species preservation" when these techniques are more broadly applied, thereby elevating (fertility) preservation from the individual to the population level.

While numerous journals, notably on reproductive medicine or assisted reproductive technology, have been publishing articles on fertility preservation, this field has been exponentially growing with the development of new strategies, patient surveys, important ethics discussions, impact of this strategy on patients and animals, etc. In the last year, almost 200 papers (original articles, reviews and editorials) with "fertility preservation" in their title were published in 50 different journals. For the scientific community and professionals working in this field, it can be quite challenging to keep updated having to perform a continuous extensive literature search.

The Journal of Fertility Preservation aims therefore to serve as a gathering point to share knowledge on all aspects of fertility preservation through publication of high-quality original papers, reviews, opinions, clinical case reports, debates, and invited editorials. As Editors-in-Chief of the Journal of Fertility Preservation, we will be dedicated to disseminate peer-reviewed basic, experimental, and clinical research related to this emerging field, including

- Strategies for fertility preservation in female and male patients.

- Stem cells associated to fertility restoration.

- Efforts to help patients affected by oncologic and nononcologic diseases safeguarding their fertility.

- Endocrine and fertility restoration.

- Counseling about fertility issues in cancer patients.

- Ethics and social issues related to fertility preservation.

- Fertility preservation strategies and protocols including aspects on gamete and tissue retrieval, storage, grafting and cryopreservation.

- Development of fertility preservation (in vitro) models.

- Fertility preservation research related to endangered animal species.

- Legal aspects of fertility preservation.

... and much more. Indeed, our ultimate goal is to be a reference platform that scientists, gynecologists, andrologists, (veterinary) surgeons, reproductive endocrinologists, embryologists, academicians, and students can turn to when publishing or getting updated on fertility preservation. 
All articles published in Journal of Fertility Preservation are open access and distributed under the Creative Commons Attribution License, which permits unrestricted use, distribution, and reproduction in any medium, provided the original work is properly cited. For more information regarding the Journal of Fertility Preservation, including our distinguished Board of Associate Editors, we invite you to visit our website (http://www.bendola.com/journals/jfp).

We also invite you to submit manuscripts to be considered for potential publication in this new peer-reviewed Journal of Fertility Preservation (JFP) and stimulate collaborating colleagues to do so.

Christiani A. Amorim and Peter E. J. Bols

Editors-in-Chief 